https://helda.helsinki.fi

\title{
Process-Tracing Methods: Foundations and Guidelines
}

\section{Ylikoski, Petri Kullervo}

2015

Ylikoski , P K 2015 , ' Process-Tracing Methods: Foundations and Guidelines ' , Contemporary Sociology, vol. 44 , no. 5 , pp. 634-635 . https://doi.org/10.1177/0094306115599351g

http://hdl.handle.net/10138/233986

https://doi.org/10.1177/0094306115599351g

acceptedVersion

Downloaded from Helda, University of Helsinki institutional repository.

This is an electronic reprint of the original article.

This reprint may differ from the original in pagination and typographic detail.

Please cite the original version. 
Process-Tracing Methods. Foundations and Guidelines, by Derek Beach and Rasmus Brun

Pedersen. Ann Arbor: The University of Michigan Press. 2013. 199 pp. paper. ISBN 9780472071890.

Petri Ylikoski

Department of Social Research

University of Helsinki

Finland

petri.ylikoski@helsinki.fi

categories: Methodology

word count: 910

\section{Process-tracing: some sensible guidelines, but still lacking foundations}

Recently political scientists have debated intensively about the case study methodology. In these debates, the so-called process-tracing methodology has acquired a prominent position as tool for making causal inferences within a single case study. In contrast to most small- $n$ case study methods that are used to make cross-case inferences about causal relationships, process-tracing methods are said to allow within-case inferences about the presence or absence of causal mechanisms. Beach and Pedersen's book is the first book-length study of process-tracing methodology, so I was curious to see what their synthesis of the political methodology has to offer for sociologists and other social scientists.

Beach and Pedersen begin by distinguishing three kinds of process-tracing. First, theory-testing process-tracing starts with an existing account of causal mechanisms and tests whether all parts of this mechanism are present in a particular case. Secondly, theory-building process-tracing is focused on discovering new causal mechanisms from case-specific empirical evidence. Finally, explainingoutcome process-tracing aims to construct an explanation for a specific historical case. This classification makes a lot of sense: the research purposes, the role of background theory, and the conclusions reached are so different that a classification like this is indispensable for making sense of process-tracing activities.

The second central element in Beach and Pedersen's account is the idea of causal mechanism. In chapter 3 and 4 the authors present their account of ontology of causal mechanisms. I found Beach and Pedersen's discussion of causation and mechanisms quite confused. Their discussions of indeterminism and ontology of causation would have benefited from reading of standard philosophical literature on these topics. Similarly, when Beach and Pedersen analyze causal mechanisms in terms of transmission of causal forces, they make unnecessary metaphysical 
commitments. Fortunately, the reader can easily skip most of these philosophical curiosities. For example, every time the authors write about causal forces, one can read them as claims about transmission of causal influence which is much more neutral (and adequate) notion for their purposes.

The third key element in Beach and Pedersen's theoretical account is a Bayesian account of confirmation which, according to them, provides "the inferential logic" for process-tracing research that brings its methodology in line with mechanism-based ontology of causation. I found this claim strange: the Bayesian logic of subjective probabilities is compatible with any ontology whatsoever. Furthermore, the most of the sensible methodological ideas they present as Bayesian are not in any way specific to Bayesian approaches and most definitely they cannot be derived from the Bayes' theorem. Luckily, one can keep their methodological discussion separate from their foundational claims.

However, their case for process-tracing methods would have been stronger without claims about the foundational role of the Bayesian confirmation theory. The problem is that the Bayesian theory tells that you have to revise your subjective confidence in the light of new evidence, but it does not really tell how to evaluate the evidence or what makes it good or objective evidence. These things are left to person's subjective assessment, the theory only requires that you revise your subjective probabilities consistently. There is of course a hope that the influence of subjective prior probabilities will be washed out once enough evidence is accumulated, but Beach and Pedersen do not even attempt to make the case that this could happen in qualitative case study research. This subjective focus leaves the door open to those who are skeptical of process-tracing methods to take Beach and Pedersen's Bayesianism as a confirmation of the ultimately subjective nature of processtracing evidence. This would be a mistaken conclusion, as I think that process-tracing perspective is important not only in qualitative case studies but also in quantitative research. Thus further work on conceptual foundations of process-tracing is still needed.

Setting aside their foundational claims, much of Beach and Pedersen's discussion of process-tracing methodology is quite useful. An example of this is their illuminating discussion of Van Evera's famous classification of the strength of predictive tests based on their ability to discriminate between competing hypotheses. They are also refreshingly clear about the limitations of processtracing methodology. As they correctly point out, most of the time within-case process-tracing needs to be supplemented both with large amounts of theoretical background knowledge, and evidence from comparative and statistical research settings. Useful is also their discussion of caseselection in Chapter 8. To my mind, this is the best chapter in the book. In contrast to other chapters 
- where Beach and Pedersen are mainly focused on specifying what makes process-tracing different from other methods - it places process-tracing in the context of larger research process. This kind of contextualization is very important for a book that is intended to be used by students.

In summary, while the book fails in its aim to provide foundations for process-tracing methodology, it still serves useful purpose by bringing together many earlier contributions to the debate about process-tracing, providing rather comprehensive account of the state of the art. For a sociologist it provides a useful summary of political science debates about process-tracing. On the negative side, the book gives an impression that is rather hastily put together. Many passages would have benefited from rewriting but also from better reading of the cited materials. All examples are from international politics, which further reduces the usability of the book in sociological methods classes. 